\title{
THE ABSENCE OF A SHORT PERIOD IN THE WN5 STAR
}

\section{EZ CANIS MAJORIS}

\author{
IGOR I. ANTOKHIN* \\ Sternberg State Astronomical Institute, \\ Universitetskij pr., 13, Moscow, 119899, Russia
}

and

\begin{abstract}
JEAN-FRANÇOIS BERTRAND*, ROBERT LAMONTAGNE* and ANTHONY F.J. MOFFAT

Département de Physique, Université de Montréal, C.P. 6128, Succ. Centre-Ville, Montréal, $Q C H 3 C 3 J 7$, and Observatoire du Mont Mégantic, Canada
\end{abstract}

Several suggestion have been put forward to explain the $3{ }^{\mathrm{d}} 766$ periodicity of EZ CMa (WR6): (i) WR+c system; (ii) rotating single WR star; (iii) pulsations (non-radial (NRP) or radial) with frequency reduced by some kind of filtering in the wind. In this paper, we report on a photometric investigation based on a long continuous observing run, in an attempt to verify whether EZ CMa does in fact show a unique periodicity. In particular, this work was motivated by the claim by Gosset \& Vreux (1987), based on the data from Lamontagne et al. (1986, hereafter LML), that EZ CMa may have a shorter period besides the one at $3{ }^{\mathrm{d}} 766$, a frequency close to but not equal to the third harmonic of the $3 .{ }^{\mathrm{d}} 766$ period.

We obtained 173 photometric observations of WR6 in a narrow-band visual filter $\left(\lambda_{c}=5140 \AA, \mathrm{FWHM}=90 \AA\right.$, pure continuum region $)$ during 57 nights, distributed in a $T=84^{\mathrm{d}}$ interval. A phase plot with the wellknown period $P=3{ }^{\mathrm{d}} 766$ is shown in Fig. 1. The power spectrum of WR$\mathrm{C} 1$ is shown in Fig.2. From Fig. 2 it is immediately clear that there is only one independent frequency in this star: $\nu_{1}=0.264 \mathrm{~d}^{-1}$. Other frequencies visible on the spectrum below $1.0 \mathrm{~d}^{-1}$ are its 2 nd and 3rd harmonics at $\nu_{2}=$ $0.530 \mathrm{~d}^{-1}\left(c f .2 \nu_{1}=0.528 \mathrm{~d}^{-1}\right)$ and $\nu_{3}=0.793 \mathrm{~d}^{-1}\left(c f .3 \nu_{1}=0.792 \mathrm{~d}^{-1}\right)$ and their aliases $1-\nu_{1}=0.736 \mathrm{~d}^{-1}, 1-\nu_{2}=0.470 \mathrm{~d}^{-1}$ and $1-\nu_{3}=0.207 \mathrm{~d}^{-1}$. Further aliases of all these occur at ever decreasing amplitude as one recedes above $\nu=1.0 \mathrm{~d}^{-1}$. Another interesting behavior is that the brightness of WR6 increased abruptly between JD 2449090 and 2449095 (data are lacking to be more precise). Points after this date are shown using different symbols in Fig. 1. This is the first example of a real brightness jump over a short time interval in WR6.

The main conclusions are:

(i) EZ CMa exhibits one period: $P=3 \cdot{ }^{\mathrm{d}} 766$. This uniqueness, along with

* Visiting Astronomer, Cerro Tololo Inter-American Observatory, National Optical Astronomy Observatories, operated by the Association of Universities for Research in Astronomy, Inc., under cooperative agreement with the National Science Foundation. 


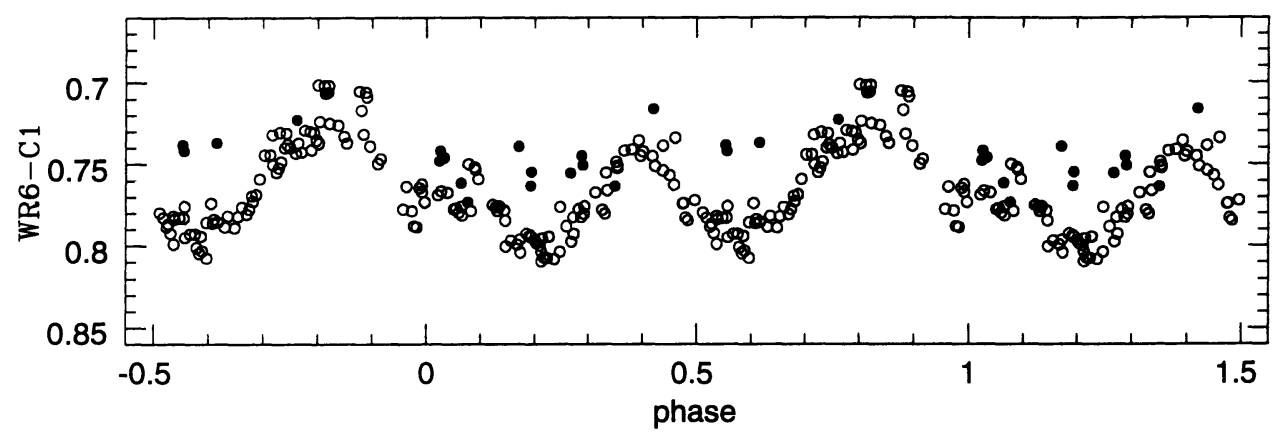

Fig. 1. Phase diagram of the WR-C1 data using the ephemeris from LML. Data after $\mathrm{JD}=2449090$ are shown with a different symbol (filled circles).

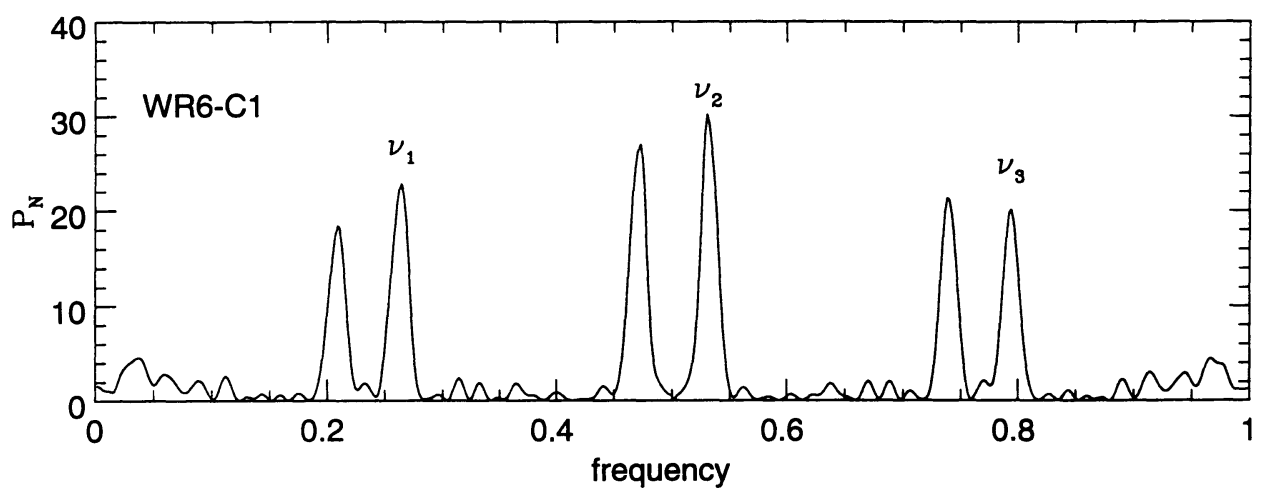

Fig. 2. Periodogram for WR6-C1 based on the present data.

the length of the period, strongly suggests that pulsations are an unlikely cause. A more likely clock is either an oblique rotator or a WR+c system. (ii) The observed light curve in 1993 Feb-May was stable over 51 days (some 13.5 cycles of the $3 .{ }^{\mathrm{d}} 766$ period), the longest coherency interval ever seen. This was abruptly interrupted by a change in form and level of the light-curve, also in an unprecedented way. This behavior might give some preference to an oblique (magnetic) rotator over a WR $+c$ system, which might be expected to be quite stable over many orbits. However, further photometric data on an even longer time scale are urgently needed to look for a tell-tale longer period (e.g., precession) as a more definite signature of a changing binary system.

\section{References}

Antokhin, I.I., Bertrand, J.-F., Lamontagne, R., Moffat, A.F.J. 1994, AJ 107, 2179

Gosset, E., Vreux, J.M. 1987, $A \varepsilon^{\prime} A$ 178, 153

Lamontagne, R., Moffat, A.F.J., Lamarre, A. 1986, AJ 91, 925 (LML) 